\title{
Tuberculous Arthritis of Knee Presenting as Bakers Cyst: A Case Report
}

\author{
Tummala Venkata Suresh Babu1, Kopuri Ravi Kiran1, Kora Deepti² \\ ${ }^{1}$ Department of Orthopaedics, Dr. PSIMS \& RF, Vijayawada, India \\ ${ }^{2}$ Department of Radiodiagnosis, Nagarjuna Superspeciality Hospitals, Vijayawada, India \\ Email: tummala36@yahoo.com
}

Received 15 January 2014; revised 14 February 2014; accepted 13 March 2014

Copyright (C) 2014 by authors and Scientific Research Publishing Inc.

This work is licensed under the Creative Commons Attribution International License (CC BY). http://creativecommons.org/licenses/by/4.0/

(c) (i) Open Access

\section{Abstract}

Tuberculosis (TB) is no longer confined to undeveloped or developing nations. Today, one third of world's population suffers from tuberculosis. Tuberculosis (TB) of the musculoskeletal system is an uncommon infection caused by tuberculous bacilli and constitutes $1 \%-5 \%$ of all forms of TB. $30 \%$ of skeletal TB involves the joints, the knee being the third most commonly affected after the spine and the hip. Knee tuberculosis commonly presents as synovitis or arthritis. Baker's cyst is a rather rare complication of knee joint tuberculosis. We report a case of a 50-year-old man with tuberculous arthritis of the right knee who presented to us as baker's cyst.

\section{Keywords}

Tuberculosis; Bakers Cyst; Loose Bodies

\section{Introduction}

A Baker cyst in the right knee-joint was demonstrated by ultrasound in a 55-year-old man with knee joint pain, effusion and popliteal swelling. Radiological examination in two planes showed degenerative changes corresponding to age with popliteal soft tissue swelling and calcified loose bodies posteriorly. Surprisingly MRI of knee demonstrated high suspicion of tubercular lesions in both femur and tibia. There was evidence of pulmonary tuberculosis radiologically but patient was asymptomatic. Culture of fluid aspirated from cyst preoperatively grew Mycobacterium tuberculosis. Cyst excision was done and sent for histopathological examination which revealed epitheloid granulomas and Langerhans giant cells. The knee-joint tuberculosis healed completely without residual damage after antitubercular treatment for one year.

\section{Case Report}

We report a case of a 55-year-old man who presented with a history of pain and swelling of right knee since 6 
months and swelling in the posterior aspect of right knee since 4 months. The patient had no cough or fever and no similar complaints in other joints. He was vaccinated with BCG as part of the national immunization programme during childhood. There were no constitutional symptoms. Patient was a known diabetic diagnosed 6 months ago and on regular treatment.

Patient could walk with an antalgic gait and had a fixed flexion deformity of $20^{\circ}$. Physical examination showed a diffuse doughy and warm swelling of right knee with a positive patellar tap. Popliteal fossa had a firm and warm swelling of about $5 \mathrm{cms}$ diameter which was located on the medial aspect of the fossa (Figure 1). Auscultation of the popliteal swelling revealed no bruit. Range of motion of the knee was from $20^{\circ}$ to $90^{\circ}$. There was no significant lymphadenopathy. Peripheral pulses and other joints were normal. Respiratory system examination revealed occasional crepitations in both lung fields. Otherwise the rest of the systemic examination was unremarkable.

On blood examination hemoglobin was $9.1 \mathrm{~g} / \mathrm{dL}$, erythrocyte sedimentation rate (ESR) was $68 \mathrm{~mm}$ in the first hour, total leukocyte count was of $8.0 \times 10^{9}$ cells/l (neutrophils, 72\%; lymphocytes, 24; monocytes, 2\%; basophils, $0.6 \%$; and eosinophils, $1.4 \%$ ) and platelet count was 142,000 cells $/ \mu \mathrm{L}$. Renal and liver function tests were within normal limits. Serum rheumatoid factor was negative. Mantoux (tuberculin) test was reactive with $15 \times$ $12 \mathrm{~mm}$ induration after 72 hours.

Plain radiography of the affected knee showed soft tissue swelling, degenerative changes in the joint and loose bodies in the posterior aspect of the knee (Figure 2(a)). Chest X-ray showed bilateral widespread fibrocavitary lesions and patchy ill defined opacities and obliteration of left CP angle due to pleural reaction (Figure 2(b)).

Utrasonography of the posterior aspect of knee joint showed a hypoechoic cystic structure in the right popliteal fossa measuring $7.7 \times 6.3 \times 3.7 \mathrm{~cm}$ with calcifications within and communicating with knee joint. There was no evidence of deep venous thrombosis (Figure 3 ).

MRI and CT scan of the knee added finer details such as synovial thickening, extensive effusion in supra patellar pouch, marrow edema at distal end of femur and proximal tibia, subarticular erosions, intact nature of the cyst (no rupture) and calcified loose bodies in the popliteal cyst (Figures 4(a) and (b)) .

Cyst was aspirated prior to excision which showed thick straw colored fluid. Aspirate was sent for gram staining, culture and sensitivity (c/s), acid-fast bacillus (AFB) smear and mycobacterial culture in Lowenstein-Jensen (L-J) medium. On Gram staining 4 - 5 pus cells/high power field were seen and bacterial c/s was negative. AFB smear was negative and L-J medium culture report had to be awaited for 3 weeks.

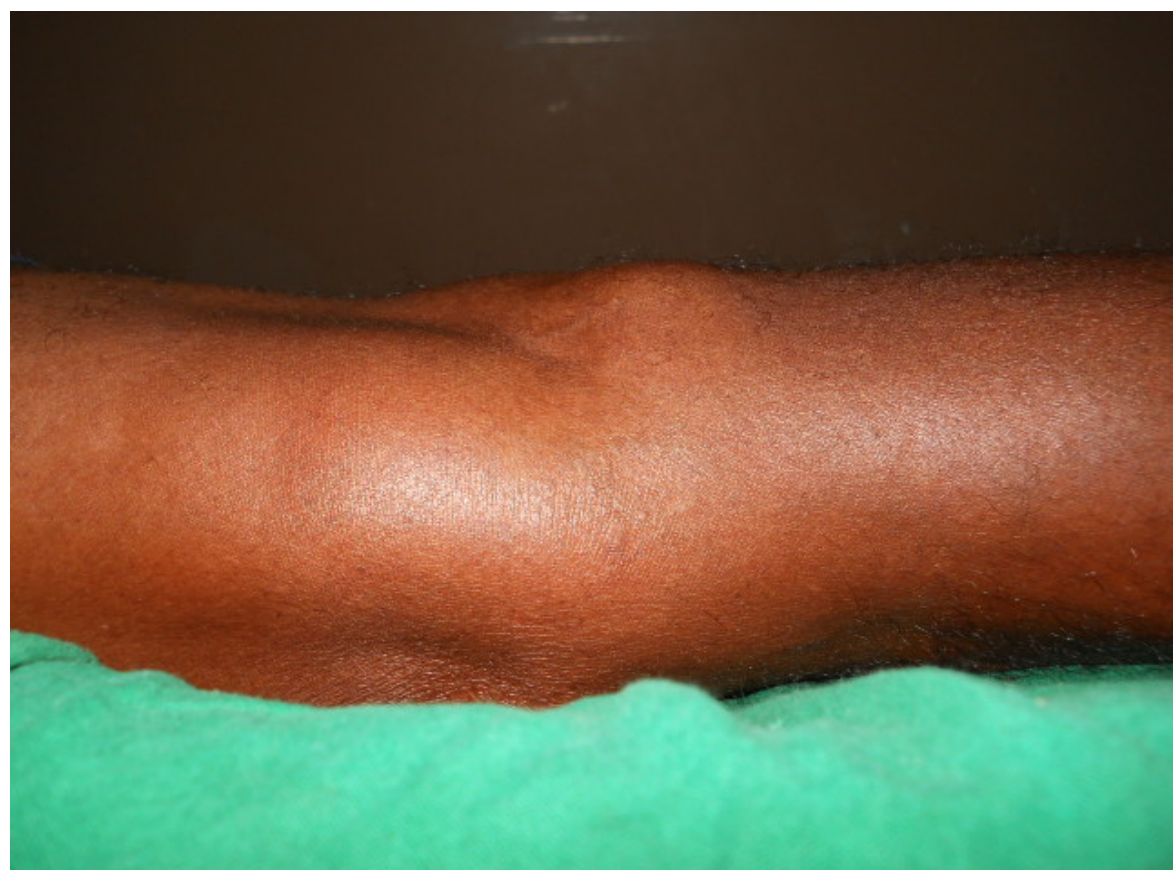

Figure 1. Clinical picture of posterior aspect of knee joint: shows a popliteal cyst. 


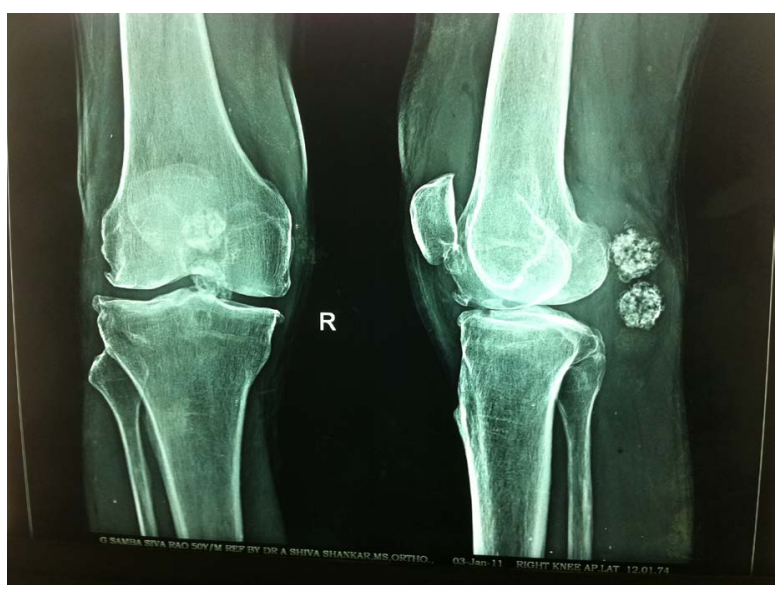

(a)

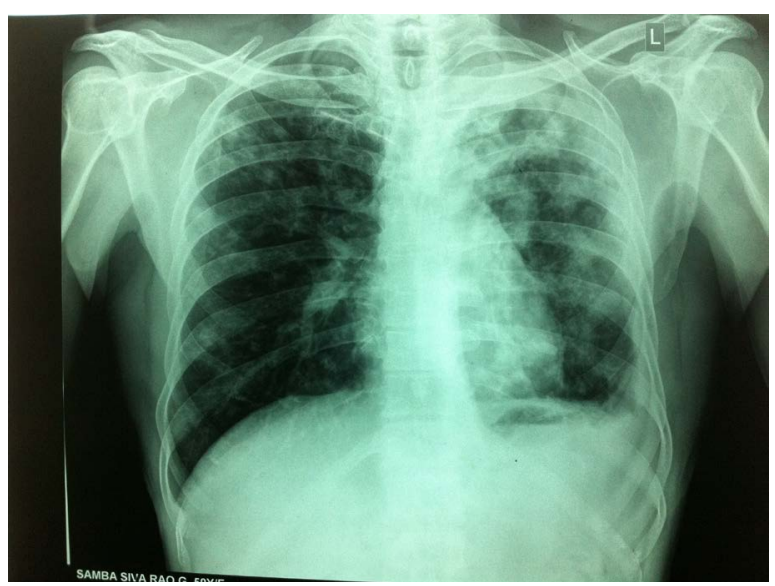

(b)

Figure 2. (a) AP/lateral view knee joint: shows arthritic changes in knee joint, loose bodies in posterior aspect of knee; (b) Chest X-ray: shows bilateral widespread fibro-cavitary lesions and patchy ill defined opacities and obliteration of left CP angle due to pleural reaction.

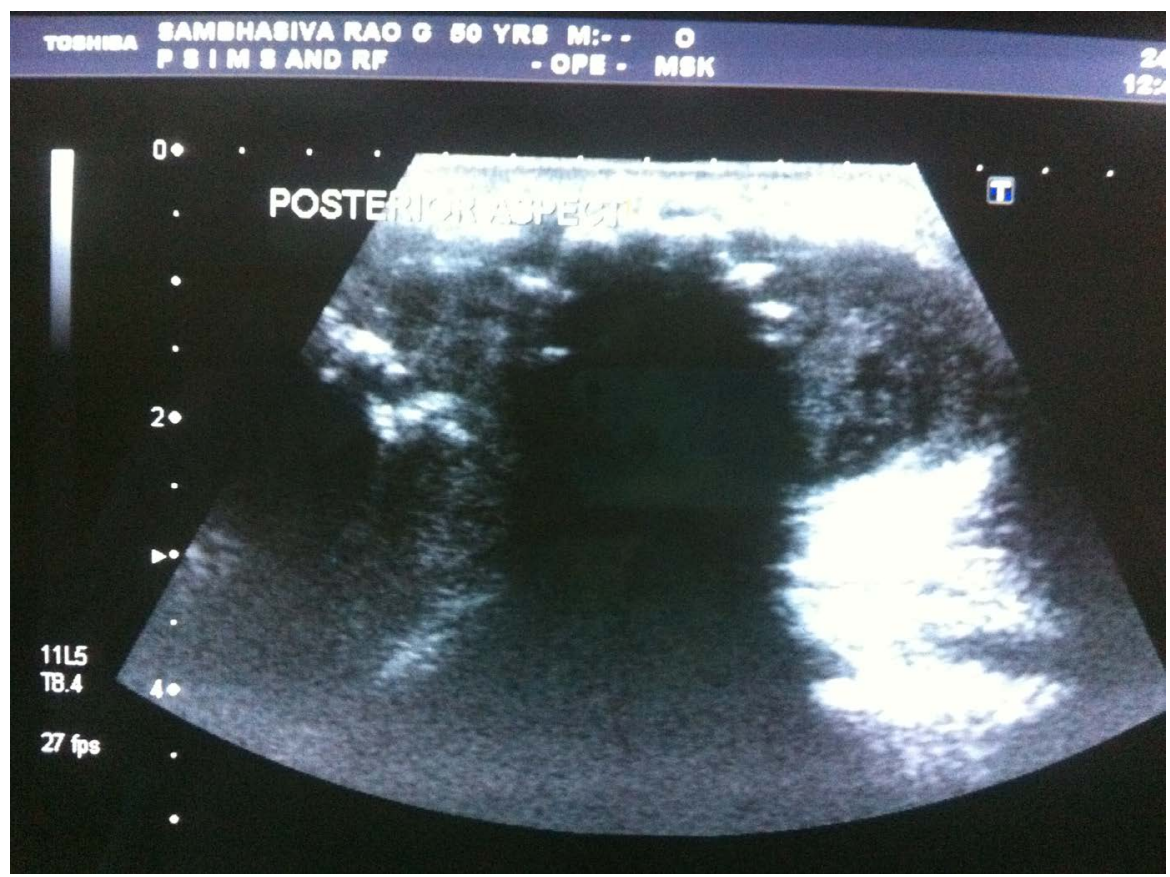

Figure 3. Utrasonography of the posterior aspect of knee: shows a hypoechoic cystic structure with calcifications within.

Dissection of the popliteal fossa facilitated exposure of a pedunculated cyst on the popliteal surface of femur with a narrow medial stalk. The cyst was related medially to the tendons of semimembranosus and semitendinosus muscles and laterally to the biceps femoris muscle. A subtotal resection of the cyst was performed evacuating $30 \mathrm{ml}$ of thick straw colored fluid and two globular loose bodies each of about $1 \mathrm{~cm}$ diameter. The histopathological examination of the cyst revealed epitheloid granulomas and Langerhans giant cells (Figure 5). At the end of 3 weeks L-J medium culture grew Mycobacterium tuberculosis confirming its tuberculosis origin.

Though symptoms subsided partially after cyst excision all the symptoms except popliteal swelling recurred within two weeks. Patient was started on anti-tuberculous treatment (ATT) postoperatively. After two months of treatment, the patient showed improvement in range of motion and reduction in the right knee swelling. ATT was continued for one year. At the end of one year patient was asymptomatic. Patient remained asymptomatic at 


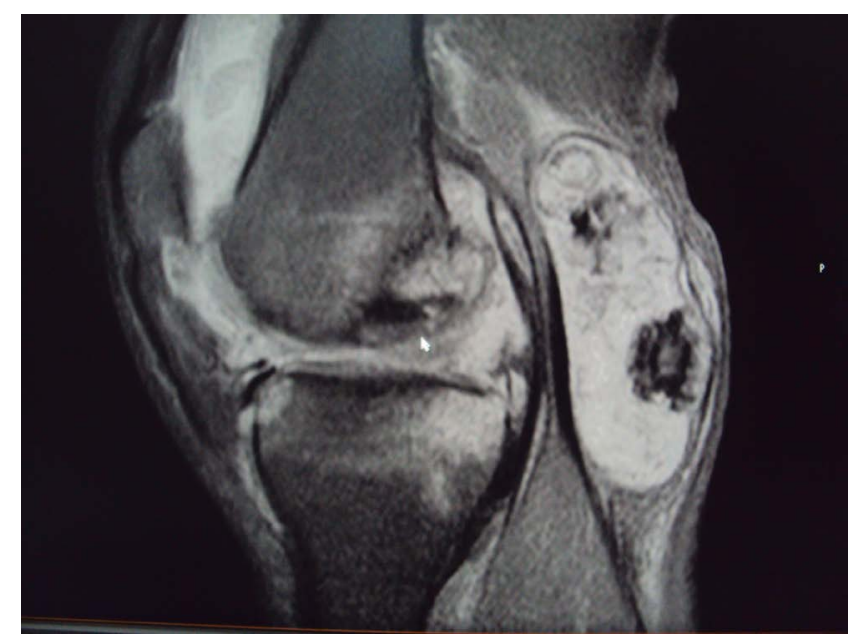

(a)

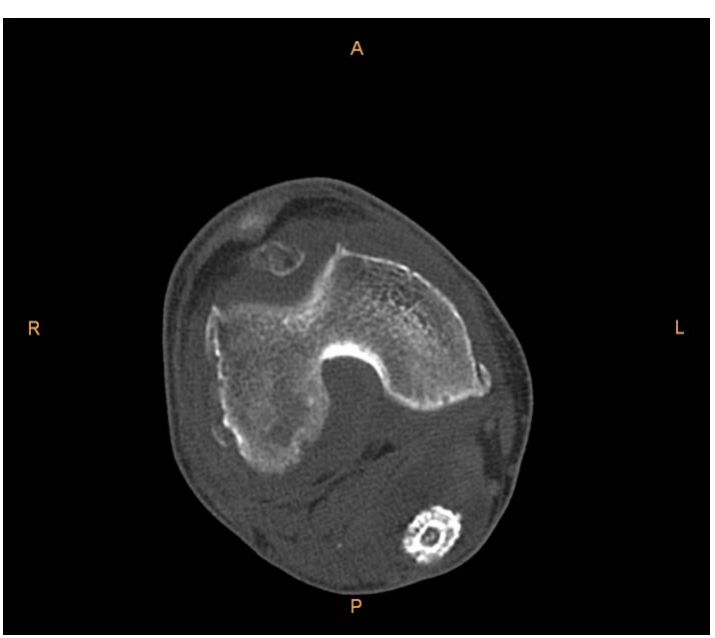

(b)

Figure 4. (a) MRI gradient $T_{2}$ saggitall view: shows extensive effusion in supra patellar pouch, marrow edema at distal end of femur and proximal tibia \& popliteal cyst with loose bodies in it; (b) CT axial view: shows calcified loose body in the popliteal cyst.

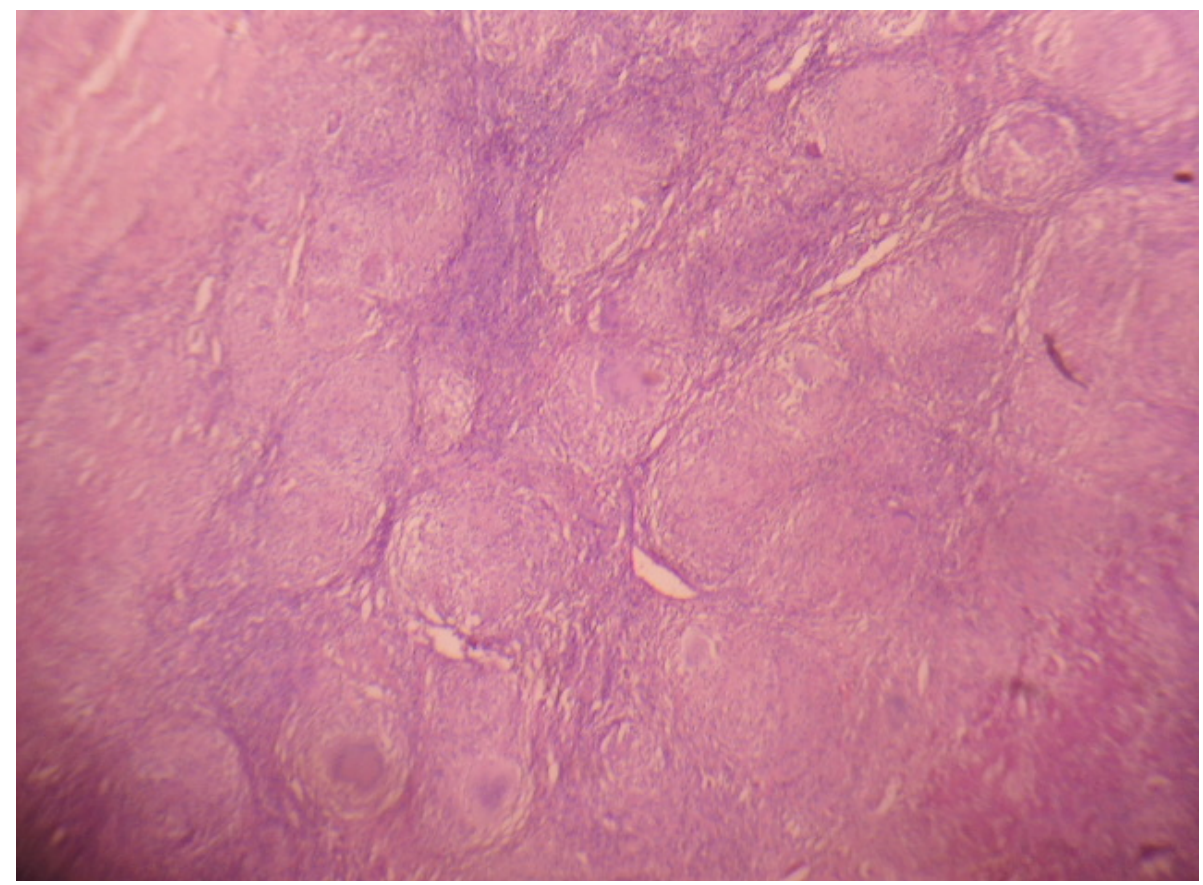

Figure 5. Microscopy: shows plenty of epitheloid granulomas and Langerhans giant cells.

the latest followup i.e., 3 yrs after primary presentation.

\section{Discussion}

Tuberculosis (TB) of the appendicular skeleton is an uncommon infection caused by Mycobacterium tuberculosis and constitutes 1\% - 3\% of all forms of TB [1]. 30\% of skeletal TB involves the joints, the knee being the third most commonly affected site [2]. Musculoskeletal involvement is through haematogenous spread often from a primary focus frequently the lungs as in this case. The incidence of skeletal TB is increasing due to the emergence of multi-drug resistant mycobacterium, increase in the number of immuno-compromised patients and the AIDS pandemic [4]. Although no age is exempted its incidence in elderly and debilitated patients is high. 
Classical presentation of knee tuberculous is chronic pain, diffuse joint swelling, local tenderness, warmth and progressive loss of function. Cold abscesses, sinuses and constitutional symptoms are also common features [3] [4]. Baker's cyst is a rather rare complication of knee joint tuberculosis [5] [6].

The Baker's cyst is a distension of the gastronemous-semimembranous bursa of the knee, which communicates with the posterior portion of the joint capsule. It usually appears as swelling in the medial aspect of the popliteal fossa secondary to pathological changes in the knee joint causing effusion. Causes are generally noninfectious knee effusion secondary to arthrosis, meniscal tears, trauma, rheumatoid arthritis and gout [7]. An infected baker's cyst is much less common and tuberculous arthritis is exceptional, in fact only few cases are described in literature till date [6].

Ultrasound will readily reveal the presence of fluid in the joint and presence of cystic structure in the popliteal fossa. It also excludes a coexisting DVT due to subjacent mass effect [8].

Early radiological findings are nonspecific and can't be differentiated from non specific arthritis. The relative preservation of joint space which is a classical feature is due to the lack of proteolytic enzymes in Mycobacterium tuberculosis [4].

Early metaphysical involvement, synovial thickening and articular changes are better demonstrated on MRI which has now become the mainstay of imaging in musculoskeletal tuberculosis [4] [9]. MRI and computerized tomography also help in outlining the baker's cyst and its contents when present; they also help in assessing rupture of the cyst [5] [8]. However imaging features are at times non-specific, but in the correct clinical context help in diagnosis of tuberculosis.

Diagnosis is often based on high suspicion index. To achieve a definitive diagnosis, it is essential to identify M. tuberculosis [10]. Bone and joint tuberculosis are however paucibacillar. Many a time Ziehl-Nielsen test is negative and it becomes necessary to wait for the Lowenstein culture results. Synovial biopsy is also an important and diagnostic method to ascertain the causative pathogen [10].

Chemotherapy remains the cornerstone of treatment; surgery is indicated in few cases which include joint debridement, synovectmy and cyst excision [2] [6].

\section{Conclusion}

To conclude our patient presented a diagnostic dilemma as he had none of the classical features of knee tuberculosis but presented with a bakers cyst (which is commonly caused by inflammatory pathology of noninfective origin). Though a positive Mantoux test did theoretically suggest the possibility of tuberculosis, this result cannot in practice be considered significant in endemic areas where most of the individuals are exposed to the infection and in previously immunized individuals. The diagnosis is finally established with a cyst fluid study and histopathology of the excised cyst, which is done as a routine in such cases. To further support the diagnosis patient had a chest $\mathrm{x}$-ray suggestive of pulmonary tuberculosis (though patient had no symptoms) and supportive MRI findings of the knee.

\section{References}

[1] Hemanth, P., Jeshil, S., Deepak, P., Manoj, S., Tufail, P. and Charles, H. (2004) Tuberculous Arthritis of the Appendicular Skeleton: MR Imaging Appearances. European Journal of Radiology, 52, 300-309. http://dx.doi.org/10.1016/j.ejrad.2003.11.003

[2] Tuli, S.M. (2004) Tuberculosis of the Skeletal System: Bone, Joints, Spine and Bursal Sheaths. 3rd Edition, Jaypee Brothers Medical Publishers, New Delhi. http://dx.doi.org/10.5005/jp/books/10993

[3] Spiegel, D.A., Singh, G.K., Banskota, A.K., et al. (2005) Tuberculosis of the Musculoskeletal System. Techniques in Orthopaedics, 20, 167-168. http://dx.doi.org/10.1097/01.bto.0000167745.24646.dc

[4] Esteban, P.L., Soriano, A., Tomas, X., et al. (2004) Tuberculous Osteomyelitis of the Knee: A Case Report. Archives of Orthopaedic Trauma Surgery, 124, 708-710. http://dx.doi.org/10.1007/s00402-004-0756-4

[5] Kulshrestha, A., Misra, R.N., Agarwal, P. and Gupta, D. (1995) Magnetic Resonance Appearance of Tuberculosis of the Knee Joint with Ruptured Baker’s Cyst. Australasian Radiology, 39, 80-83. http://dx.doi.org/10.1111/j.1440-1673.1995.tb00240.x

[6] Bianco, G., Paris, A., Venditti, M., Calderini, C., Anzivino, C. and Serra, P. (2001) Popliteal (Baker’s) Cyst in a Patient with Tubercular Arthritis-Report of a Case and Review of the Literature. Recenti Progressi in Medicina, 92, 663-666. 
[7] Weinstein, S.L. and Buckwalter, J.A. (1994) Turek’s Orthopaedics: Principles and Their Application. 5th Edition, JB Lippincott, Philadelphia.

[8] Radford, D.M., Schuh, M.E., Nambisan, R.N. and Karakousis, C.P. (1993) Pseudo-Tumor of the Calf. European Journal of Surgical Oncology, 19, 300-301.

[9] De Vuyst, D., Vanhoenacker, F., Gielen, J., Bernaerts, A. and de Schepper, A.M. (2003) Imaging Features of Musculoskeletal Tuberculosis. European Journal of Radiology, 13, 1809-1819. http://dx.doi.org/10.1007/s00330-002-1609-6

[10] Hoffman, E.B., Allin, J., Campbell, J.A.B., et al. (2002) Tuberculosis of the Knee. Clinical Orthopaedics and Related Research, 398, 100-106. http://dx.doi.org/10.1097/00003086-200205000-00014 